\title{
Purinergic Signalling special issue on "Purinergic Signalling in the Inner Ear"; a commentary by Professor Geoffrey Burnstock, Editor-in-Chief
}

\author{
Geoffrey Burnstock
}

Published online: 20 July 2010

(C) Springer Science+Business Media B.V. 2010

This special edition-"Purinergic Signalling in the Inner Ear"- assembled under the steerage of Guest Editors Gary Housley and Jonathan Gale, extends the recent series of issues that have highlighted emerging themes of purinergic signalling. This special edition reflects the breadth of understanding of the role of extracellular nucleoside and nucleotide signalling in the cochlea. The inner ear has revealed fascinating processes related to the development and function of hearing and balance with broad relevance to other systems. The ten fully peer-reviewed papers are provided by research leaders who have made sustained contributions to this field and have accepted the invitation to contribute highlights of their current research focus to this special edition.

The issue opens with papers describing the dynamics of ATP release mechanisms and related inter-cellular synchronous communication between the sensory and supporting cells and neurons. These studies resolve connexin hemi- channel mediated ATP release, controlled by P2X and P2Y receptors, contributing to the establishment of auditory neurotransmission, and effector pathways, including $\mathrm{Ca}^{2+}$ and nitric oxide second messenger signalling, that sustain the function and integrity of the cochlea. Subsequent papers provide new data on the breadth of $\mathrm{P} 1$ and $\mathrm{P} 2$ receptor and NTPDase expression in the cochlea and investigate the dynamics of these systems with regard to hearing function with ageing and noise-stress. Of particular note is the final paper, by Vlajkovic et al, which provides an exciting glimpse at the translational potential of the field. This study reports what may be the first effective treatment of hearing loss after noise trauma; systemic treatment of rats with the A1 receptor agonist (adenosine amine congener-ADAC) significantly reduced noise-induced hearing loss. As this issue moves from immediate on-line access, to print, it is pleasing to report that this exciting finding has already attracted considerable media attention.

G. Burnstock $(\bowtie)$

Autonomic Neuroscience Centre Royal Free Campus,

University College Medical School,

Rowland Hill Street,

London NW3 2PF, UK

e-mail: g.burnstock@ucl.ac.uk 